\title{
Zentrale IT-Services der FH Bonn-Rhein-Sieg
}

\section{EINLEITUNG}

\subsection{Entwicklung der Fachhochschule}

Die Fachhochschule Bonn-Rhein-Sieg wurde zum 01.01. 1995 mit dem Hauptsitz in Sankt Augustin und zunächst einer Abteilung in Rheinbach sowie ab dem 01.09.2002 einer weiteren Abteilung in Hennef gegründet. Die Entwicklung der neuen Fachhochschule belegt folgende Übersicht:

\section{5}

Studiengänge

Studienanfänger

Personal

2 Studiengänge
58 Studierende
22 hauptamtlich Beschäftigte ${ }^{1}$

1999

Studiengänge

Studienanfänger

Studierende insgesamt

Personal

\section{4}

Studiengänge

Studienanfänger

Studierende insgesamt

Personal

\section{Studiengänge}

492 Studienanfänger

1289 Studierende

126 hauptamtlich Beschäftigte ${ }^{2}$

Der Aufbau der Fachhochschule ist damit noch nicht abgeschlossen.

Die Fachhochschule gliedert sich organisatorisch in

- Fachbereich 01 Wirtschaft Sankt Augustin,

- Fachbereich 02 Angewandte Informatik,

- Fachbereich 03 Elektrotechnik, Maschinenbau, Technikjournalismus,

- Fachbereich 04 Wirtschaft Rheinbach,

- Fachbereich 05 Angewandte Naturwissenschaften,

- Fachbereich 06 Sozialversicherung,

- Institut für interdisziplinäre Studien ${ }^{4}$,

- Institut für Existenzgründung und Mittelstandsförderung ${ }^{5}$,

- Bibliothek,

- Sprachenzentrum und

- Verwaltung.

In den Jahren 1995 bis 1999 war die Fachhochschule an den Standorten Sankt Augustin und Rheinbach in Provisorien un-

\footnotetext{
Einschließlich Teilzeitbeschäftigte.

Einschließlich Teilzeitbeschäftigte.

3 Einschließlich Teilzeitbeschäftigte.

4 Formale Gründung steht noch aus.

5 Formale Gründung steht noch aus.
}

tergebracht. An beiden Standorten bezogen die Hochschulangehörigen im August 1999 die neuen Hochschulgebäude. Beide Gebäude wurden im Februar 2005 um Erweiterungsbauten ergänzt. Dem vom Hauptverband der Berufsgenossenschaften (HVBG) finanzierte Studiengang Sozialversicherung stehen Räume im Gebäude des HVBG am Standort Hennef zur Verfügung.

\subsection{Zuständigkeit für die IT-Services}

Der Gründungsrektor übertrug im Jahre 1997 der Hochschulverwaltung offiziell die an den Fachhochschulen üblicherweise von den Datenverarbeitungszentralen (DVZ) wahrzunehmenden Aufgaben der Informations- und Kommunikationstechnik. Damit wurden folgende zentralen IT-Services mit dem IT-Service der Hochschulverwaltung an der Fachhochschule gebündelt:

- zentrale Netzwerkinfrastruktur,

- zentrale Serverdienste (Mail, DNS, Proxy, WVVW uvm.),

- zentrale Hörsaaltechnik,

- Telefonie,

- Hochschul-Datenbanksysteme (HIS, MACH, Buisy),

- Fileserver (Verwaltung) und

- PC der Verwaltung sowie deren Peripherie.

Auf diese Weise konnten Synergieeffekte genutzt und eine erhebliche Kostenersparnis erzielt werden.

\subsection{Entwicklung der IT-Infrastruktur bis zum Jahre 2002}

Nach Gründung der Fachhochschule stand der zügige Aufbau des Lehrbetriebs (siehe Nr. 1.1) im Vordergrund. Im Zuge der kontinuierlichen Weiterentwicklung des Studienangebotes „wuchs“ die IT-Umgebung. Es entstand eine IT-Infrastruktur, die zwar flexibel an die wachsenden Anforderungen der Gliederungen (Fachbereiche, zentrale Einrichtungen und Verwaltung) angepasst werden konnte, sich aber im Laufe der Zeit zu einer Art "Gemischtwarenladen" mit allen nachteiligen Auswirkungen entwickelte.

Im Zuge der Neubauplanung - Bezug im Jahre 1999 - wurde das Netzwerk der Fachhochschule erstmals systematisch strukturiert. Merkmale des neuen Konzeptes für die zentrale ITInfrastruktur waren:

- Ein „voll-geswitchtes“ 100 Mbit/s Netzwerk mit 18 eingesetzten per Glasfaserring verbundenen CISCO-Switches, die ca. 2000 Ports versorgten. 
- Der Backbone basierte auf ATM-Technologie und stellte eine Bandbreite von $622 \mathrm{Mbit} / \mathrm{s}$ zur Verfügung.

- Die Endgeräte waren über eine strukturierte CAT5 Vollverkabelung angebunden.

- Das Netzwerk des Standortes Rheinbach war über eine Richtfunkstrecke mit einer Bandbreite von $155 \mathrm{Mbit} / \mathrm{s}$ an die Zentrale in Sankt Augustin angekoppelt. Trotz der räumlichen Trennung nahmen die Nutzer der Infrastruktur nur ein Netzwerk wahr.

- Der DFN-Verein stellte über das Wissenschaftsnetz G-Win die $34 \mathrm{Mbit} / \mathrm{s}$ Internetanbindung bereit.

- Zur Abwicklung der Telefoniedienste wurde eine Tenovis-Telefonanlage mit ca. 500 Nebenstellen eingesetzt. Die ISDNTelefonanlage bot neben dem eigentlichen Telefondienst für jeden Nutzer eine Anrufbeantworter- und eine Fax-Funktion.

- Der betreute Serverpark umfasste insgesamt 45 Systeme, die in der Mehrzahl aus dedizierten Einzelsystemen bestanden. Mehrere Raid-Festplattensubsysteme (Raid-5 Level) versorgten diese Systeme mit der benötigten Speicherkapazität. Als Hardware für die Serversysteme wurde größtenteils „No-Name Standard -PC-Technik“ verwendet. Außerdem wurden noch einige HP und SUN-Systeme eingesetzt.

- Eine zentrale Backuplösung existierte nicht. Die unterschiedlichen Systeme wurden in verschiedenen Teilbackups über lokale Bandlaufwerke gesichert.

- Die Betriebsystempalette umfasste die gesamte Bandbreite der gängigen Produkte von Windows NT über Linux (diverse Derivate) bis hin zu Unix (SUN, HP).

Den Studierenden wurde an beiden Standorten ein Selbstbedienungsterminal bereitgestellt. Über diese Terminals hatten die Studierenden die Möglichkeit, diverse Vorgänge zeitsparend, örtlich und zeitlich unabhängig vom Studierendensekretariat zu erledigen. Zur Benutzung des Terminals berechtigte eine Chipkarte (Studierendenausweis).

Zur PC-Ausstattung der Verwaltung wurden im Jahre 2000 sechzig PC-Systeme geleast. Bei den einzelnen PCs handelte es um Individualsysteme, die an die Anforderungen des jeweiligen Anwenders angepasst waren. Die Einzelsystem-Konfiguration wurde über „Images“ gesichert.

In den zehn zentralen Hörsälen der Fachhochschule wurden Systeme mit einer identischen Multimedia-Grundausstattung installiert. Über diese Medientische (inkl. PC, DVD, Video etc.) konnten die Vortragenden die Beamer, die Mikrophontechnik sowie die Beleuchtungs- und Verdunklungseinrichtungen der Hörsäle zentral steuern.

\section{AUSGANGSLAGE FÜR DIE NEUSTRUKTURIERUNG DER IT-INFRASTRUKTUR IM JAHRE 2003}

\subsection{Entwicklung der Fachhochschule}

Neben der unter Nr. 1.1 im Überblick beschriebenen qualitativen und quantitativen Entwicklung der Fachhochschule zeichneten sich im Jahre 2002 konkret folgende Maßnahmen ab, die sich unmittelbar auf die Anforderungen an die IT-Services auswirkten und eine grundlegende Überarbeitung des IT-Infrastruktur Konzeptes erforderten:

- Für die Standorte Sankt Augustin und Rheinbach wurden Erweiterungsbauten geplant, die eine Erweiterung der luKInfrastruktur notwendig machten.
- Der Studiengang Sozialversicherung nahm am dritten Standort der Fachhochschule in Hennef den Studienbetrieb auf. Dieser Standort musste in die luK-Infrastruktur eingebunden werden.

- Die Studierendenzahl der Fachhochschule überschritt deutlich das geplante Ausbauziel von 2.500 Studienplätzen.

\subsection{Schwachstellenanalyse}

Eine Schwachstellenanalyse offenbarte, dass die IT-Infrastruktur der weiteren Entwicklung nicht lange gewachsen sein wird. Die Ursachen dieser nachstehend aufgeführten Schwachstellen ergaben sich teilweise aus

- der zwischenzeitlich eingetretenen technologischen Entwicklung im IT-Bereich,

- der fehlenden Standardisierung und

- sonstigen Unzulänglichkeiten.

\subsubsection{Technologische Entwicklung}

- Viele Hardwarekomponenten (Server und Netzwerk) wurden durch die Hersteller gekündigt, so beispielsweise Windows NT durch die Firma Microsoft.

- Für andere Komponenten wie beispielsweise die Richtfunkanlage waren Ersatzteile nicht mehr lieferbar.

- Die aktiven Netzwerkkomponenten (Switches) hatten ihre Ausbaugrenze erreicht.

- Die ATM-Technologie, auf der der Backbone der Hochschule basierte, wurde von der Firma CISCO gekündigt. Dies hatte Auswirkungen auf die Richtfunkstrecke, die als Folge einer Migration ebenfalls mit neuer Technik bestückt werden musste.

- Die PC-Ausstattung der Verwaltung musste erneuert werden. Der Leasingvertrag hat sich wegen zum Teil ungenügender Supportleistungen nicht bewährt. Das „Sichern“ von Images erwies sich als sehr aufwändig in der Administration. Für minimale Änderungen mussten die Images jedes Mal überarbeitet werden

- Die Medientechnik in den Hörsälen war veraltet und bedurfte einer Erneuerung.

\subsubsection{Fehlende Standardisierung}

- Die Server-Infrastruktur bestand aus einem "Server-Zoo“ mit Hardware, die ebenfalls das Ende der Lebensdauer erreichte. Durch den Einsatz verschiedener „No-Name-Produkte" existierten ebenso viele verschiedene Hardwareverträge, die die Handhabung unübersichtlich gestalteten.

- Das Teilbackupkonzept hat sich als äußert aufwendig in der Administration erwiesen. Eine umfangreiche Windowsmigration war notwendig.

\subsubsection{Sonstige Unzulänglichkeiten}

- Das Netzwerk war bis dahin nur durch rudimentäre Routerfilter gesichert. Die Fachhochschule benötigte dringend einen umfassenden Schutz per Firewall.

- Die Serviceverträge liefen aus.

- Die Dreiteilung der Aufgaben zur Netzwerktechnik, Telefonie und der PC-Technik stellte sich als nachteilig heraus. Es gab Kompetenzprobleme bei der Überschneidung von Dienstleistungen. 
- Die Telefonanlage hatte ihre Ausbaugrenzen erreicht. Eine einfache Erweiterung über Leitungsanschlüsse war nicht möglich. An allen Standorten waren weitere Anlagenmodule erforderlich.

- Der PC-Leasingvertrag lief zum 01.04.04, die Netzwerkund Telefoniewartungsverträge zum 31.12.2004 aus.

\section{NEUSTRUKTURIERUNG DER IT-INFRASTRUKTUR}

Aufgrund der unter Nr. 2 aufgezeigten Entwicklung und der Schwachstellenanalyse entschied sich die Fachhochschule in Abstimmung mit dem Wissenschafts- und dem Finanzministerium im Jahre 2003 zu einer umfassenden Erneuerung der ITInfrastruktur.

\subsection{Zielsetzungen}

\subsubsection{Leistungs-/Kapazitätserweiterung aller luK-Systeme}

Vorrangiges Ziel war die Leistungssteigerung aller Systeme und langfristig ein Betrieb ohne kostspielige Folgeanschaffungen. Soweit möglich sollten in allen Systembereichen Standardkomponenten von Markenherstellern eingesetzt werden.

\subsubsection{Reduzierung der Betriebskosten}

Die Betriebskosten sollten durch eine Vereinfachung der Administration bzw. des Administrationsmanagements für alle Systemkomponenten (Netzwerk, Firewall, Telefonie, Server, PC) und die Vermeidung von "Insel-Know-how" reduziert werden.

\subsubsection{Reduzierung der Wartungskosten}

Trotz der angestrebten Leistungssteigerung für alle Systemkomponenten sollten die Reaktionszeiten im Störungsfall gedehnt und somit die Wartungskosten reduziert werden. Dabei war insbesondere an Maßnahmen zur Ausfallsicherheit (Redundanzen) gedacht. Ferner sollte der einzukaufende Service „aus-einer-Hand“ kommen.

\subsubsection{Netzwerksicherheit (Firewall)}

Das Netz sollte insbesondere durch den Einsatz von Firewalls vor Angriffen von außen und innen geschützt und damit das zwischenzeitlich erarbeitete Sicherheitskonzept der Fachhochschule umgesetzt werden.

\subsubsection{Ausfallsicherheit (Hardware)}

Die Systeme sollten - soweit möglich und sinnvoll - Redundant ausgelegt werden.

\subsubsection{Flexibilität/Mobilität}

Unter Berücksichtigung des Datenschutzes wurde ein weltweiter, systemunabhängiger Zugriff angestrebt, um damit unter anderem die Voraussetzungen zur Einrichtung von Home-Arbeitsplätzen zu realisieren.

\subsubsection{Zukunftssicherheit/Integrationsfähigkeit}

Das Gesamtsystem sollte - soweit erkennbar - den Anforderungen der nächsten fünf bis zehn Jahre entsprechen. Spätere Erweiterungen oder Ergänzungen sollten problemlos integrierbar sein.

\subsection{Umsetzung}

Zur Umsetzung der zu vor definierten Zielanforderungen wurde im Februar 2004 das Migrationsprojekt „luk-ReNew“ eingeleitet und nach nur 12 Monaten erfolgreich abgeschlossen. In dieser Zeit wurde der IT-Betrieb weitgehend aufrechterhalten. Das Gesamtprojekt unterteile sich in 14 Teilprojekte:

- Migration des LANs

- Erneuerung Richtfunkverbindung

- Einführung der Firewall

- Erneuerung der Telefonie (VoIP)

- Migration der Servertechnik

- Einführung eines SAN

- Erneuerung des Backupsystems

- Einführen von Client/Servertechnologie in der Verwaltung (Citrix)

- Windows 2003/Active Directory-Migration

- Realisierung von Home-Arbeitsplätzen

- Notstromversorgung der Server- und Verteilerräume

- Bau- und Klimatisierungsmaßnahmen in den Server- und Verteilerräumen

- Einführung eines WLAN

- IT-Anbindung der Neubauten

\subsection{Netzwerk}

\subsubsection{Beschreibung}

Kern des „voll-geswitchten“ CISCO-Netzwerks ist der 1 Gbit/s Backbone. Die Standorte Sankt Augustin und Rheinbach sind über eine Richtfunkstrecke in einem transparenten Netzwerk verbunden (Anhänge A und B). Die „Core-Switches“ des Backbones sind redundant ausgelegt. Die Arbeitsplatzrechner sind über eine strukturierte CAT7 Verkabelung mit $100 \mathrm{Mbit} / \mathrm{s}$, die Server teilweise mit $1 \mathrm{Gbit} / \mathrm{s}$ angebunden. Die logischen Netzwerke basieren auf der VLAN-Technik von CISCO. Derzeit sind ca. 130 VLANs definiert. Gesicherte externe Zugriffe werden mittels VPN umgesetzt. Dabei stehen zwei Alternativen zur Verfügung.

- Alternative 1 „Site to Site VPN“

Die externe Gegenstelle benutzt eine konfigurierte Firewall (Netscreen 5GT/5XP). Es wird eine dedizierte verschlüsselte Internetverbindung aufgebaut. Mittels Firewallregeln werden die gewünschten Zonen freigeschaltet.

- Alternative 2 „Client to Site VPN“

Dazu wird auf dem externen Client ein Netzwerkconnector installiert. Die Verbindung zur Hochschule erfolgt über das Internet auf einen VPN SSL Router.

Der Standort Hennef ist über eine 2Mbit/s Standleitung angebunden und stellt für die Hochschule im Prinzip ein „Extranet“ dar, da der HVBG das dortige Netzwerk weiterhin administriert.

Das hochschulweite WLAN bietet mit etwa 80 verteilte CISCO Accesspoints eine nahezu vollständige räumliche Verfügbarkeit des Netzes an den Standorten Sankt Augustin und Rheinbach. 
Die Netztopologie ist in der beiliegenden Skizze (Anhang A) dargestellt.

\subsubsection{Zielerreichung}

Sämtliche aktiven Komponenten des gesamten Backbones sind erneuert, die Portanzahl der Switches verdoppelt. Die Bandbreite des Backbones beträgt nunmehr $1 \mathrm{Gbit} / \mathrm{s}$, bisher 622 Mbit/s. Die Neubauten an den Standorten Sankt Augustin und Rheinbach sind problemlos in das Gesamtnetz integriert. Der Backbone ist weitgehend mit Redundanzen (Anhang A) versehen. Jeder der insgesamt 18 Etagenswitches ist über eine redundante Wegeführung physikalisch an den Backbone angebunden. Die möglichen Portgeschwindigkeiten liegen bei 10/ 100/1000, vorher bei 10/100. Weiterhin bieten die neuen Switches nun auch die Möglichkeit des so genannten Power-overEthernets. Damit können Endgeräte (z.B. Telefon oder WLANAccesspoints) aus dem Datennetzwerk mit Strom versorgt werden.

Kabelungebundene Schnittstellen (WLAN) ergänzen das Netzwerk der Fachhochschule.

Die Nettokapazität der Datenübertragungsrate auf der Richtfunkverbindung beträgt nach dem Technikumstieg ca. 150 Mbit/ s, vorher etwa $80 \mathrm{Mbit} / \mathrm{s}$.

Innerhalb des gesamten Backbones wird nur noch das Ethernet-Protokoll verwendet. Die ATM-Technologie ist vollständig abgelöst. Daher ist kein spezielles ATM-Know-how mehr notwendig. Der konsequente Einsatz von CISCO-Technologie vereinfacht die Administration und das Management des Netzes.

Die teilweise redundant ausgelegten Komponenten bieten die Möglichkeit, die Wartungsanforderungen gröber zu staffeln. Die Reaktionslevels sind an den jeweiligen Grad der Wichtigkeit der Komponenten angepasst. Core-Komponenten werden im Störfall unmittelbar ersetzt, bei alle anderen Komponenten wird eine längere Ausfallzeit in Kauf genommen.

Die Ausfallsicherheit der Richtfunkstrecke wird über die beiden Amtsleitungen der Telekom, mit denen ein Notbetrieb möglich ist, gewährleistet. Die beiden Server- und alle Verteilerräume sind mit unterbrechungsfreien Stromversorgungen gegen Stromausfälle gesichert und ausreichend klimatisiert.

Der Backbone ist so ausgelegt, das er jederzeit ohne größeren Aufwand um zusätzliche Komponenten erweitert werden kann. Die stringent standardisierte Kommunikationstechnologien und der Verzicht auf proprietäre Technologien gewährleistet eine hohe Integrationsfähigkeit des Netzwerkes.

Durch die konsequente Nutzung von Standardtechnologien und die Berücksichtigung von skalierbaren Komponenten kann von einer zukunftssicheren Investition ausgegangen werden. Alle eingesetzten Komponenten unterstützen bereits den Standard „IPv6“.

\subsection{Firewall}

\subsubsection{Beschreibung}

Im Zuge der Neuorganisation der luk-Infrastruktur wurde die seit langem geforderte Firewall installiert. Die Netscreen Fire- wall ist in der Lage, die verschieden internen Netzbereiche (Mitarbeiternetz, Studierendennetz, Poolbereich uvm.) gegenseitig abzuschotten und kontrolliert auch den Übergang zum Internet. Sie erfüllt dabei alle im Sicherheitskonzept der Hochschule geforderten Anwendungsbereiche (Anhänge $E$ und F). Derzeit werden etwa 130 Netzbereiche mit einem umfassenden Regelwerk von der Firewall abgedeckt. Durch die Definition von „virtuellen" Firewalls ist es möglich, diese dezentral zu administrieren. Damit kann jede Gliederung eigene Netzbereiche kontrollieren und ein spezielles Regelwerk definieren. Es sind momen$\tan 9$ virtuelle Firewallsysteme im Einsatz.

\subsubsection{Zielerreichung}

Es können bis zu 1024 Netzbereiche definiert und kontrolliert werden. Erweiterungen sind jederzeit realisierbar. Die Firewall ist redundant ausgelegt. Beim Ausfall der aktiven Firewall übernimmt automatisch und ohne Unterbrechung die zweite Firewall den Betrieb.

Administrationsaufgaben (beispielsweise das Einspielen von Updates, Patches etc.) können ohne Störung des laufenden Betriebs vorgenommen werden.

Die Firewall bietet die Möglichkeit, VPNs zu terminieren, gesicherte Zugriffe von beliebigen externen Standorten auf Hochschuldaten zuzulassen und so unter anderem Home-Arbeitsplatzes zu realisieren.

Die Firewall unterstützt IPv6 und ist somit für kommende Anwendungen gerüstet.

\subsection{Servertechnik}

\subsubsection{Beschreibung}

Eines der Hauptziele zur Neugestaltung der luK-Infrastruktur war die Serverkonsolidierung. Die vormals 45 Server-Hardware-Systeme wurden auf 12 Systeme (IBM X445) reduziert. Auf diesen Servern wird die Virtualisierungssoftware VMWare eingesetzt über die sich virtuelle Server definiert lassen. Pro Serverhardware sind ohne Performanceverlust bis zu 12 virtuelle Systeme abgebildet. Diese Technik ist unabhängig von der Betriebssystem Plattform, so dass der Einsatz von Windows-, Unix- oder Linux Systemen nicht eingeschränkt ist. Alle Server greifen auf ein Zentrales Speichersystem, das so genannte Storage Aera Network (SAN) mit ca. 1,4 TByte Nettospeicherkapazität zu. Das SAN ist eine der wenigen Komponenten, die einen „Single point of failure“ im Gesamtsystem darstellen und daher hochredundant ausgelegt ist. Das betrifft die physikalischen Datenverbindungen sowie das Speichersystem selbst.

Ein zentrales Backupsystem, dessen Kernstück eine Tape-Library (40 Bänder) mit Backuproboter ist, sichert die Daten.

Am Standort Sankt Augustin sind zwei Serverräume mit jeweils 6 Serversystemen in verschiedenen Gebäudeteilen untergebracht. Das SAN und das Backupsystem sind ebenfalls räumlich getrennt. Die Komponenten der beiden Serverräume sind über redundant ausgelegte Gbit-Switches verbunden.

Das zentrale Management der Serverkomponenten besteht aus zwei Teilbereichen. Mit dem Managementsystem „IBM-Di- 
rector" lassen sich alle zwölf Server-Maschinen von einer Konsole (Remote Zugriff, beliebiger Standort) bis auf „BIOS“Ebene konfigurieren und administrieren. Über das „Virtuell Center" von VMWare kann auf alle 55 virtuellen Server zugegriffen und Veränderungen im Betriebssystem vorgenommen werden.

\subsubsection{Zielerreichung}

Durch den Einsatz von VMWare stehen einfache und flexible Serversysteme zur Verfügung. Dadurch ist es möglich, die meisten der mehr als 50 virtuellen Serversysteme redundant auszulegen. Ein fehlerhaftes System kann schnell wieder hergestellt werden. Zudem ist es möglich, die virtuellen Server auf den verschiedenen ,physikalischen“ Servern beliebig zu portieren.

Durch die bereits installierten virtuellen Serversysteme sind „Templates“ vorhanden, die die Umsetzung eines virtuellen Basissystems in wenigen Minuten ermöglichen. Diese Option verleiht der eingesetzten Servertechnik eine Flexibilität, die mit der herkömmlichen Serverhardware nicht erreichbar ist.

In der momentanen Ausbaustufe ist ein reibungsloser Betrieb von etwa $72(6 \times 2)$ Servern möglich. Unter Ausschöpfen der Lastgrenzen wäre diese Zahl erweiterbar. Auch das Einfügen zusätzlicher Serverhardware ist jeder Zeit möglich. Die weitestgehende Betriebssystemunabhängigkeit gewährleistet bisher nicht vorgesehene Anwendungsmöglichkeiten.

Durch den Einsatz der zwölf identischen IBM X445-Maschinen und die Implementierung von VMWare konnte die gewünschte Standardisierung realisiert werden. Die Management-Systeme "IBM-Director" und „Virtuell Center" garantieren vereinfachte Administration und effektives Management. Die Administration der Systeme ist standortunabhängig und lässt sich von jedem beliebigen hochschulinternen oder -externen Arbeitsplatz durchführen.

Im Wartungsfall können die virtuellen Server ohne Unterbrechung des produktiven Betriebs auf ein Ersatzsystem portiert werden. Hardwarefehler werden den Administratoren per Email mitgeteilt, so dass eine unmittelbare Reaktion zur Behebung der Störung möglich ist. Servicelevels zum Austausch fehlerhafter Hardware wurden so gesenkt.

Das SAN verfügt über redundant auslegte Kontroller. Das RaidSystem ist so dimensioniert, dass bis zu fünf Festplatten gleichzeitig ausfallen können, ohne dass der Betrieb des Speichersystems beeinflusst wird.

Die Sicherung der Serverdaten wird mit einem zentralen Backupsystem realisiert.

\subsection{Telefonie}

\subsubsection{Beschreibung}

Die neue Telefonietechnik besteht aus einem System von Servern, Routern, Gateways und IP-basierten Endgeräten (Anhang $\mathrm{C}$ ). Ein Telefonendgerät ist damit ein kleiner Computer, der an jeder Stelle des Netzwerkes der Fachhochschule angeschlossen werden kann, ohne dieses Telefon jedes Mal neu verkabeln oder konfigurieren zu müssen.
Die Steuersysteme der IP-Telefonie (Callmanager) sind redundant ausgelegt und jeweils auf die Standorte Sankt Augustin und Rheinbach verteilt.

Die Anbindung an das externe Telefonnetz der Telekom erfolgt über Gateways. In Sankt Augustin stehen dafür zwei S2m-Anschlüsse, in Rheinbach steht ein S2m-Anschluss zur Verfügung. Durch die Kopplung der Callmanager ist es somit möglich, 90 externe Gespräche gleichzeitig zu führen.

Als Endgeräte stehen drei unterschiedliche Typen zur Verfügung, die alle mit einer Fax-/Voice-Funktion ausgestattet und über das UMS-System in den Mailserver eingebunden sind. Überwiegend eingesetzt sind das „Komforttelefon 7940“ in den Büros und das „Standardtelefon 7902“ in den Laboren und Besprechungsräumen.

\subsubsection{Zielerreichung}

Der Umstieg von einer klassischen ISDN-Telefonanlage hin zur VolP-Technologie war wesentlich kostengünstiger als die Erweiterung der zuvor eingesetzten ISDN-Telefonanlage. Weiterer Vorteile ist die Einpassung der VoIP-Technologie in das umzusetzende, vollgeswitchte CISCO-Netzwerk (Homogenisierung der luk-Infrastruktur).

Die Anzahl der Nebenstellen wurde von 500 auf 600 erhöht. Die Neubauten an den Standorten Sankt Augustin und Rheinbach sind nahtlos in die Telefonie eingebunden. Das VoIP-System ist für Endgeräte beliebig erweiterbar. Faktisch gibt es keine Ausbaugrenze wie beim Vorgängersystem von TENOVIS. Beim auch temporären Wechsel des Arbeitsplatzes kann das Endgerät mitgenommen werden, ohne dass eine Umkonfiguration notwendig ist.

Die Betriebskosten wurden erheblich reduziert, da das Telefonienetz integraler Bestandteil des Datennetzes ist. Ein klassisches Telefonnetz mit eigener Verkabelung, eigener Administration und eigenem Management existiert an der Hochschule nicht mehr. Auf klassisches Telefonie-Spezial-Know-how kann daher gänzlich verzichtet werden.

Alle zum Telefonbetrieb benötigten Netzwerkkomponeten (Gateways, router etc.) sind von einem Hersteller (CISCO). Die Softwareapplikationen (Call Manager, UMS, Gebührenerfassung) werden auf Windowsplattformen betrieben. Hardwaregrundlage sind IBM-Standardsysteme. Der Callmanager bietet offene Softwareschnittstellen für benutzerorientierte Funktionen. Auch bei der Gebührenabrechnung konnten Schnittstellen zur Einbindung in Workflows genutzt werden. Die Bearbeitungszeit des Abrechnungsvorgangs wurde damit um $80 \%$ der ursprünglichen Arbeitszeit reduziert.

Die Wartungskosten konnten durch die Integration des Telefonie- in das Datennetz erheblich gesenkt werden. Der Bereich Telefonie ist nun Bestandteil des Gesamtwartungsvertrages Netzwerk. Schnittstellen fielen weg und das Idealziel „Serviceaus-einer Hand" konnte erreicht werden.

Die zentralen Komponenten des VolP-Systems wurden an den Standorten Sankt Augustin und Rheinbach installiert. Beim Ausfall einer Komponente an einem Standort übernimmt die jeweils andere den Betrieb. Da an beiden Standorten jeweils ein „Amtskopf“ eingerichtet wurde, ist die Hochschule beim Ausfall 
eines Amtskopfes über den anderen Amtskopf weiterhin mit dem externen Telefonnetz verbunden.

Das Feature „Least-cost-routing“ kann über die Standorte Sankt Augustin und Rheinbach genutzt werden.

Unter Einbeziehung der VPN-Technologie ist die Fachhochschule in der Lage, vollwertige IT-Büroarbeitplätze inkl. Bürotelefonanschluß als Home-Arbeitsplätze zur Verfügung zu stellen. Da VolP den Kommunikationsstandard für Datennetze (TCP/IP) als Transportmedium benutzt, ist das System optimal ins Gesamtsystem eingebunden. Schnittstellen zwischen IPbasierten Endgeräten sind leicht umzusetzen.

VoIP stellt den neuen Übertragungsstandard in der Telefonie der Zukunft dar. Telefonieanbieter wie z.B. die Telekom AG planen die Umrüstung des bestehenden Telefoniebackbones auf VolP. Eine Ausdehnung der bisher „intern“ genutzten VolPTechnik auf die externe Telefonie ist problemlos umsetzbar.

\subsection{Hörsaaltechnik}

Die PC-Hardware in allen Medientischen wurde ausgetauscht und auf den neusten Stand der Technik gebracht. Identische PC-Systeme minimieren den Administrationsaufwand.

\subsection{Verwaltungs-IT}

\subsubsection{Beschreibung}

Für die Hochschulverwaltung wird ein Server based Computing (Citrix) eingesetzt, auf dem sämtliche von den Nutzern benötigte Anwendungssoftware (Office, HIS, Mach etc) installiert ist. Die Thinclients (Wyse) sind trotz „einfacher“ Technik wesentlich performanter als die vorher eingesetzten PC-Systeme. Sowohl die redundant ausgelegten Citrix-Server wie auch die Thinclients werden „Remote“ administriert.

Bei Störungen an den Thinclients, die sich nicht „Remote“ beheben lassen, wird die komplette Hardware durch vorinstallierte Reservegeräte ausgetauscht. Die Arbeitsplätze sind in solchen Fällen in kürzester Zeit wieder voll funktionsfähig.

Administrationsaufgaben wie beispielsweise das Einspielen von Patches werden im laufenden Betrieb vorgenommen. Während der Administrationsphase wird das System aus der Serverfarm genommen und die Funktionen von einem Ersatzgerät übernommen. Nach Ausführen der Systemänderungen und ausgiebigen Tests übernimmt das Originalsystem wieder den produktiven Betrieb. Die Änderungen werden anschließend am Reservesystem ebenfalls durchgeführt.

\subsubsection{Zielerreichung}

Die „Turnschuh-Administration“ der PC-Systeme wird durch die einfache und flexible Nutzung der Fernwartungsmöglichkeiten bis auf ein Minimum reduziert.

Durch die doppelte Auslegung der Citrix-Serverhardware sind keine Systemabschaltungen, beispielsweise für Administrationsaufgaben, notwendig. Neuinstallationen von Anwendungs- software auf dem Citrix-Server stehen unmittelbar nach Freigabe allen Nutzern zur Verfügung.

Die Installation der Anwendungsprogramme auf dem zentralen Citrix-Server vereinfacht auch das Lizenzmanagement.

Auf spezielle Supportverträge wird verzichtet.

Server based Computing und Thinclienttechnologie sind höchst flexibel. Durch den Einsatz von Standardhardware kann jeder Nutzer an jedem beliebigen Arbeitsplatz arbeiten. Arbeitsplatzveränderungen (bereits bewährt beim Umzug in die Erweiterungsbauten an beiden Standorte) stellen kein großes Problem dar. Im Zusammenspiel mit VoIP-Technik und VPN kann der Nutzer seine „elektronische Büroausstattung“ mit nach Hause nehmen.

Im Zuge, der sich allgemein durchsetzenden Zentralisierungsbestrebungen bietet „Server based Computing“ eine kostengünstige, skalierbare, administrationsfreundliche und ortsunabhängige Alternative zu der klassischen Server/PC-Technik dar.

\section{ZUSAMMENFASSUNG UND AUSBLICK}

Sämtliche Zielanforderungen des Projektes luK-ReNew werden über das gesamte Spektrum der durchgeführten Migration erreicht. Die bisherigen Erfahrungen im produktiven Einsatz der Systeme bestätigen, dass trotz der erheblichen Leistungssteigerungen aller Teilsysteme die Administrations- und Managementaufgaben verringert werden konnten. Ein Indiz dafür ist die Neubesetzung nur einer von zwei frei gewordenen Administratorstellen.

Die Systemumgebung ist so ausgelegt, dass die Fachhochschule flexibel auf neue Anforderungen reagieren kann. Folgende Szenarien sind denkbar:

\subsection{Kurzfristige Anforderungen}

\subsubsection{Support für Gründerfirmen}

In den Erweiterungsbauten an den Standorten Sankt Augustin und Rheinbach sind unter anderem auch die Firmen des Gründerzentrums unterbracht. Die Betreiber des Gründerzentrums haben sich dazu entschlossen, die gleiche Telefonie- und Netzwerktechnik einzusetzen wie die Fachhochschulen. Durch Mitnutzung der hochschuleigenen Infrastruktur werden zum Vorteil beider Seiten Synergien erzielten.

\subsubsection{Home-Arbeitsplätze}

Die technischen Voraussetzungen zur einfachen Anbindung von Home-Arbeitsplätzen sind realisiert. Der Modellversuch Telearbeit in der Hochschulverwaltung wurde inzwischen eingeleitet.

\subsubsection{UNI@home}

Mit der T-Com wurde vereinbart, eine Juniper-VPN-Lösung zu installieren. Damit werden „Client to VPN“-Verbindungen über die Firewall und der gesicherte Zugriffe der Studierenden auf 
dedizierte Bereiche des Hochschulnetzwerks über das Regelwerk der Firewall möglich.

\subsubsection{Credo}

Die Fachhochschule könnte an das Gbit/s-Extranets „Credo“, das den Wissenschaftseinrichtigen in Raum Köln/Bonn von der Firma Netcologne zur Verfügung gestellt wird, angebunden werden.

\subsubsection{SAN-Erweiterung}

Derzeit wird über eine Erweiterung des SAN nachgedacht. Unklar ist noch, ob die bestehende Technik aufgerüstet werden soll oder ob eine Kapazitätserhöhung durch ein „virtuelles“ SAN die sinnvollere weil flexiblere Alternative ist.

\subsection{Langfristige Anforderungen:}

\subsubsection{Dias und Telefonie}

Es ist geplant, die Gebührenabrechnung unter anderem mit dem Ziel zu automatisieren, die Abrechnungen über DIAS ${ }^{6} \mathrm{di}-$

6 Ein hochschulintern entwickeltes Work-flow-System mit vielfältigen Anwendungsmöglichkeiten. rekt an jedem Arbeiterplatz zu bearbeiten. Des Weiteren ist geplant, Telefonfunktionalitäten (Kurzwahl, Telefonbuch etc.) auf den ThinClients zur Verfügung zu stellen.

\subsubsection{Backup}

Nach einer Erweiterung der Backupkapazitäten könnte man anderen Hochschulen die Auslagerung ihres Backups ermöglichen.

\subsubsection{Citrixerweiterung}

Eine Ausweitung des Server-based-Computing ist ohne großen Aufwand möglich. Sollten sich außer der Hochschulverwaltung weitere Gliederungen der Fachhochschule zum Einsatz dieser Technik entschließen, kann ohne großen Aufwand eine entsprechende Kapazitätserweiterung vorgenommen werden.

\subsubsection{VolP-Kopplung}

Weitere VolP-Kopplungen (siehe Gründerfirmen) oder Kooperationen mit anderen Hochschulen sind möglich. Gemeinsames „Least-cost-routing“ ist einfach zu realisieren.

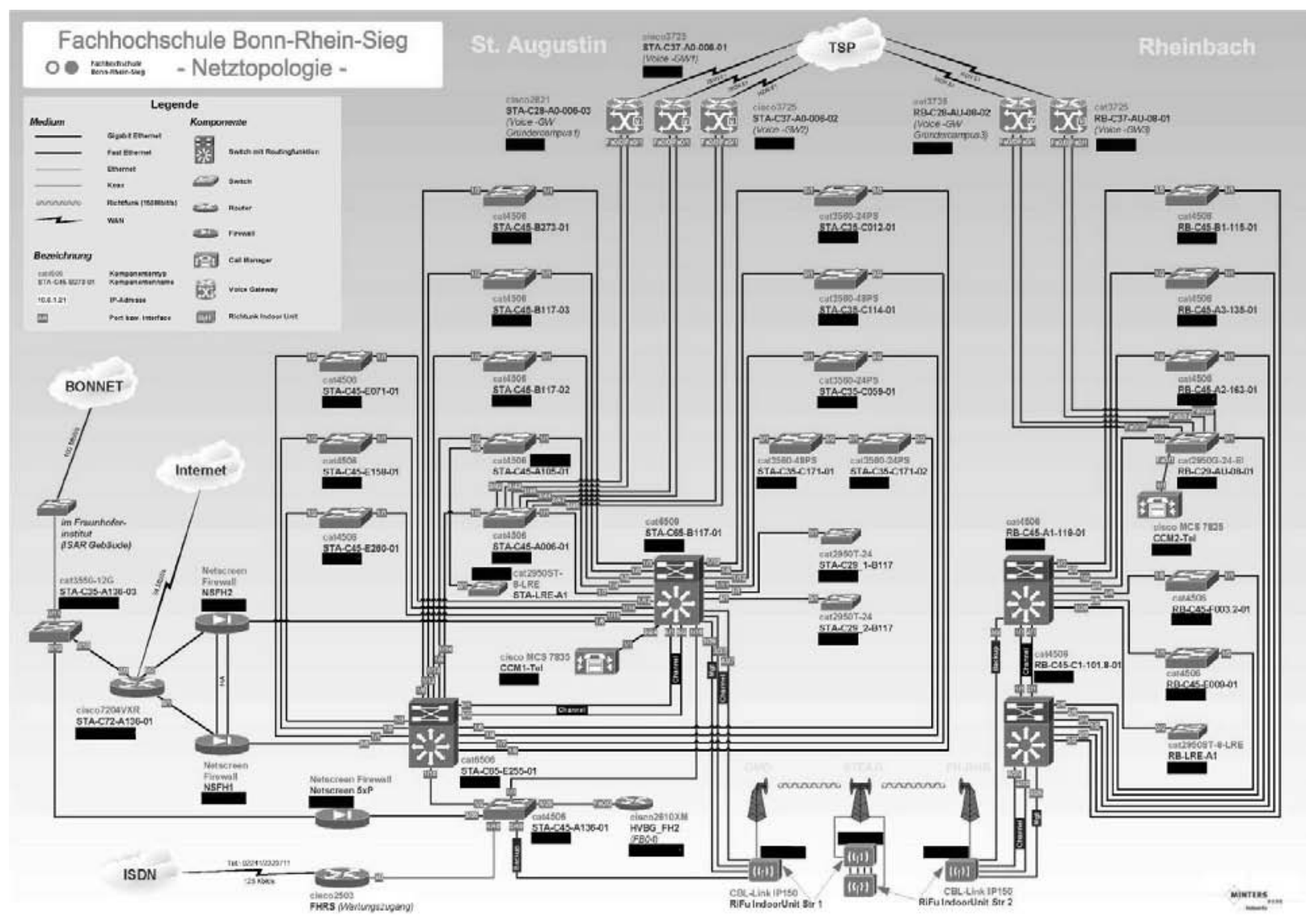

Anh. A Netztopologie 


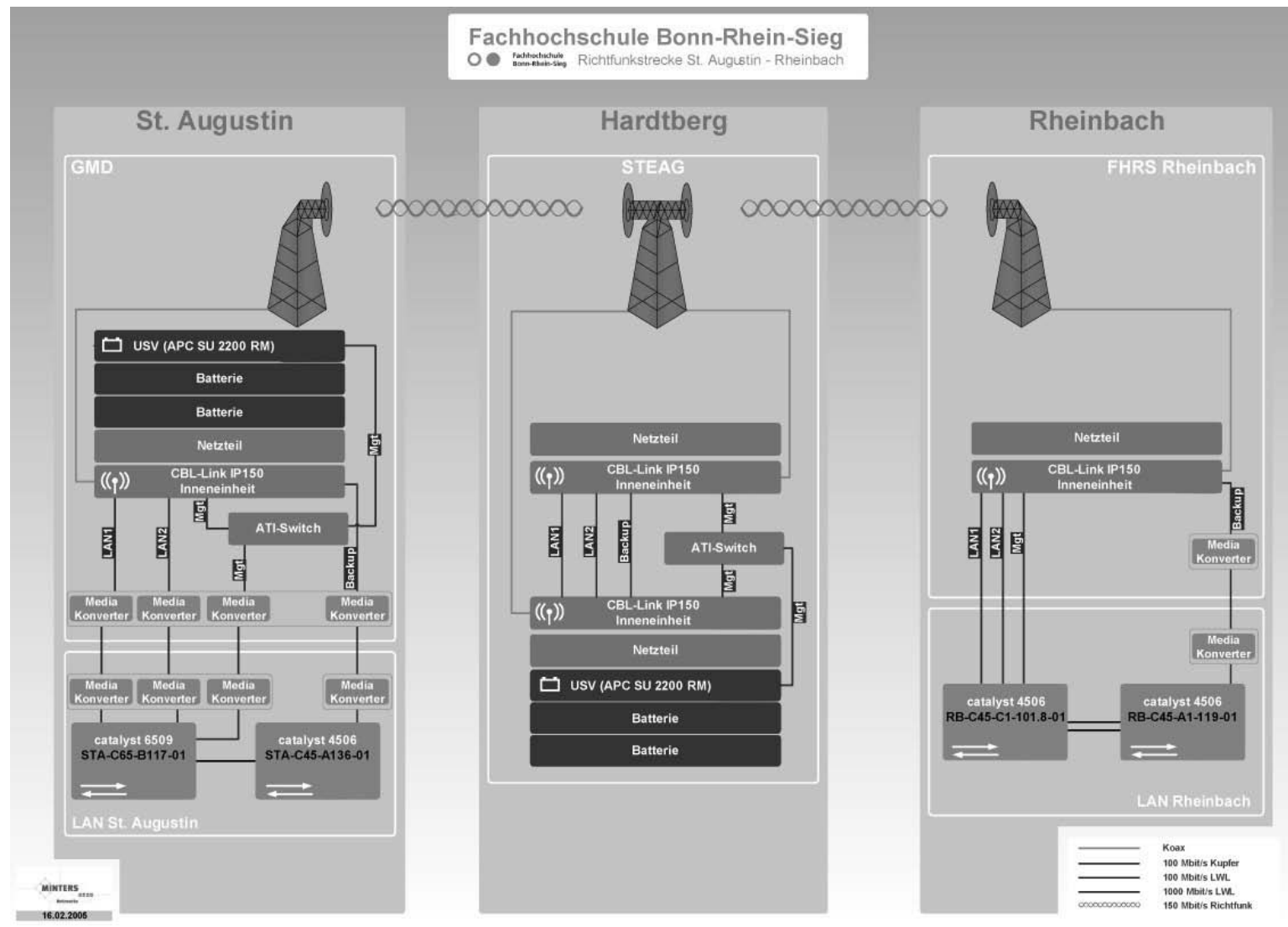

Anh. B Richtfunk

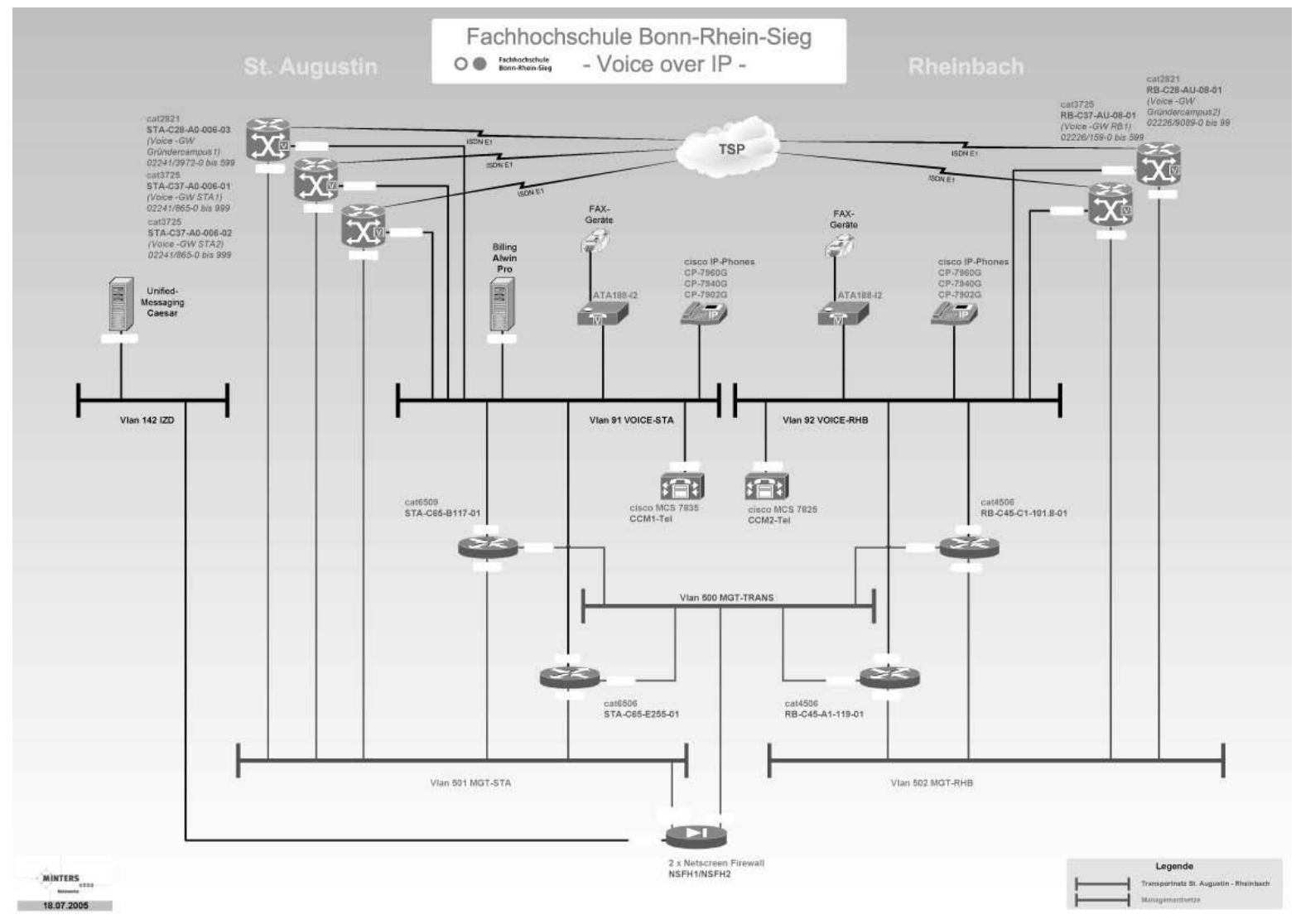

Anh. C VolP 


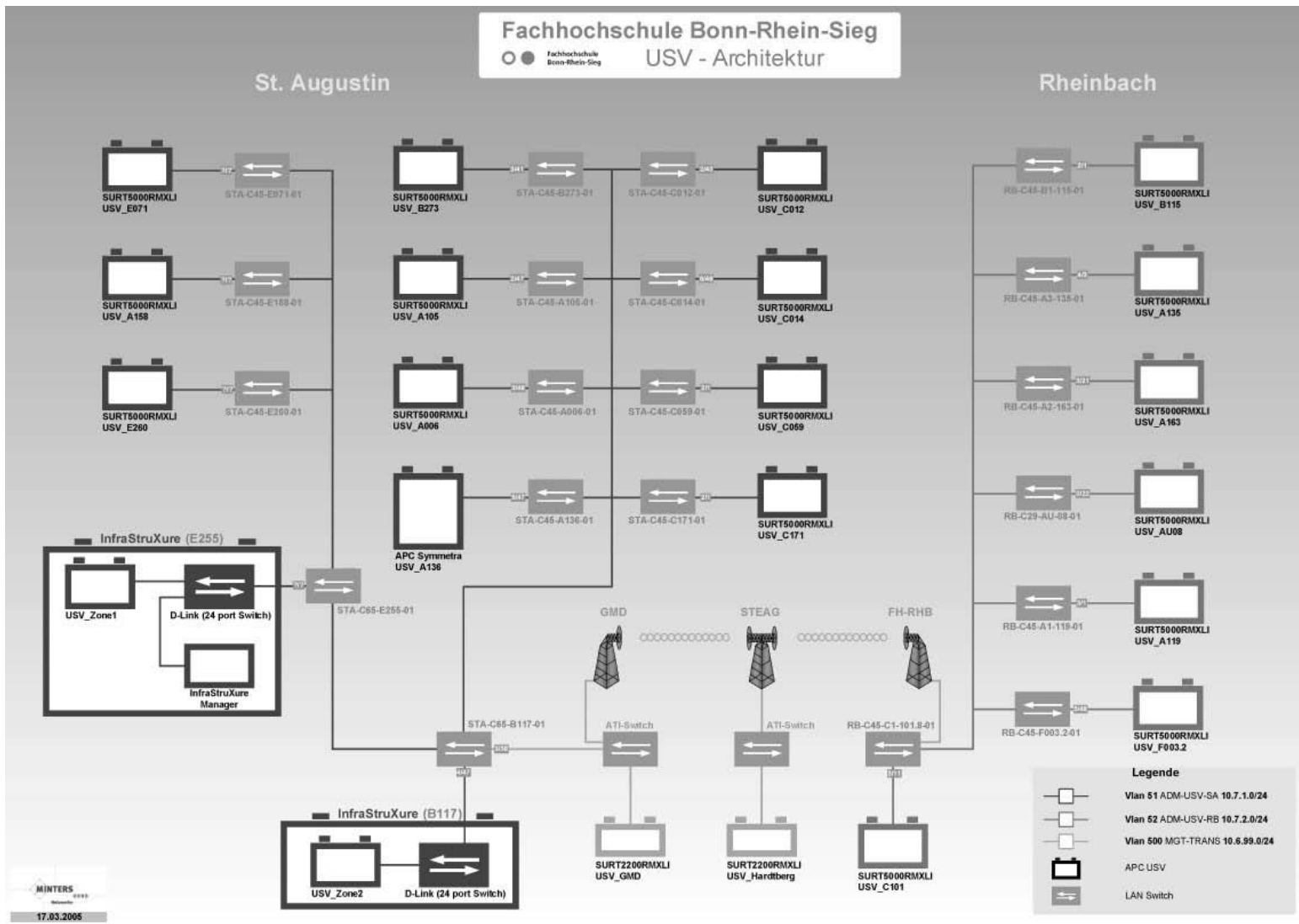

Anh. D USV

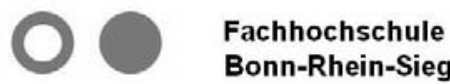 \\ Firewall- Struktur an der $\mathrm{FH}$}

\begin{tabular}{|l|}
\hline Zuständigkeit \\
$\square$ DFN-Verein \\
$\begin{array}{l}\text { luK-SB der Gliederungen } \\
\text { bzW. Dekan(in) / Leiter(in) }\end{array}$ \\
$\square$ zentrale(r) luK-SB \\
\hline
\end{tabular}

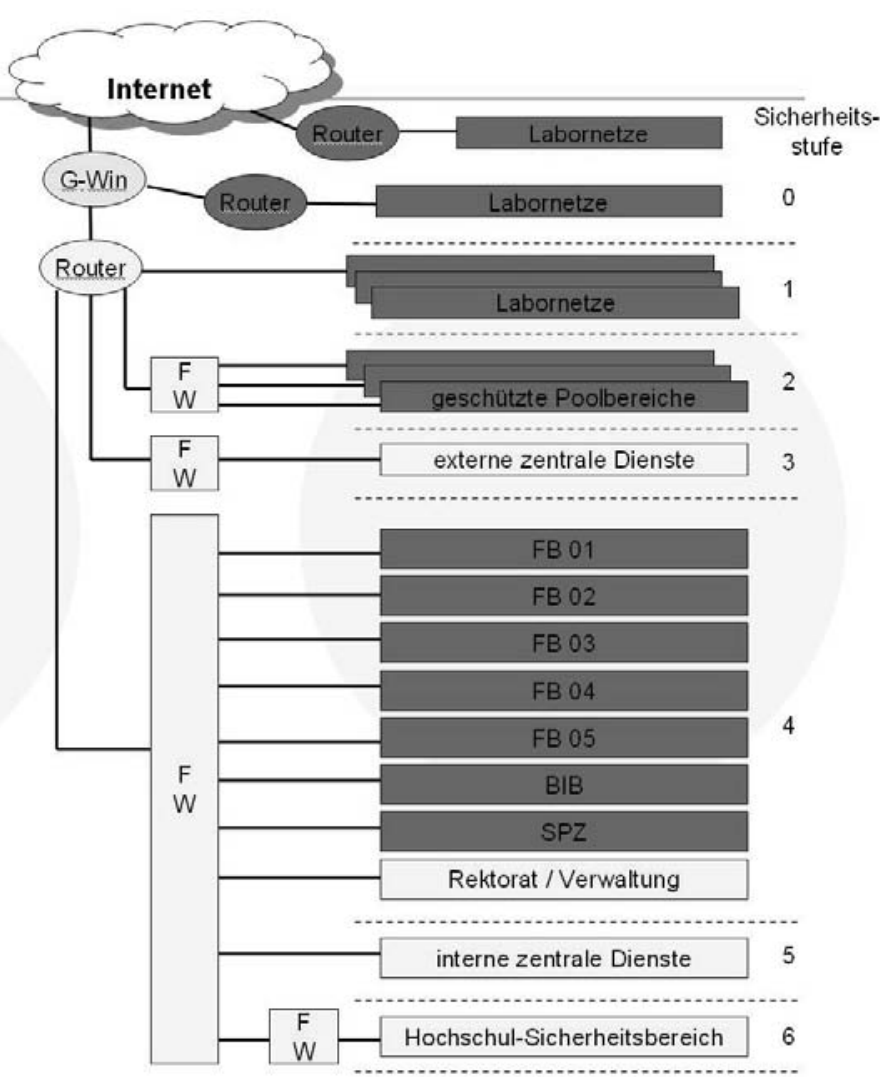

Anh. E Firewallstruktur 


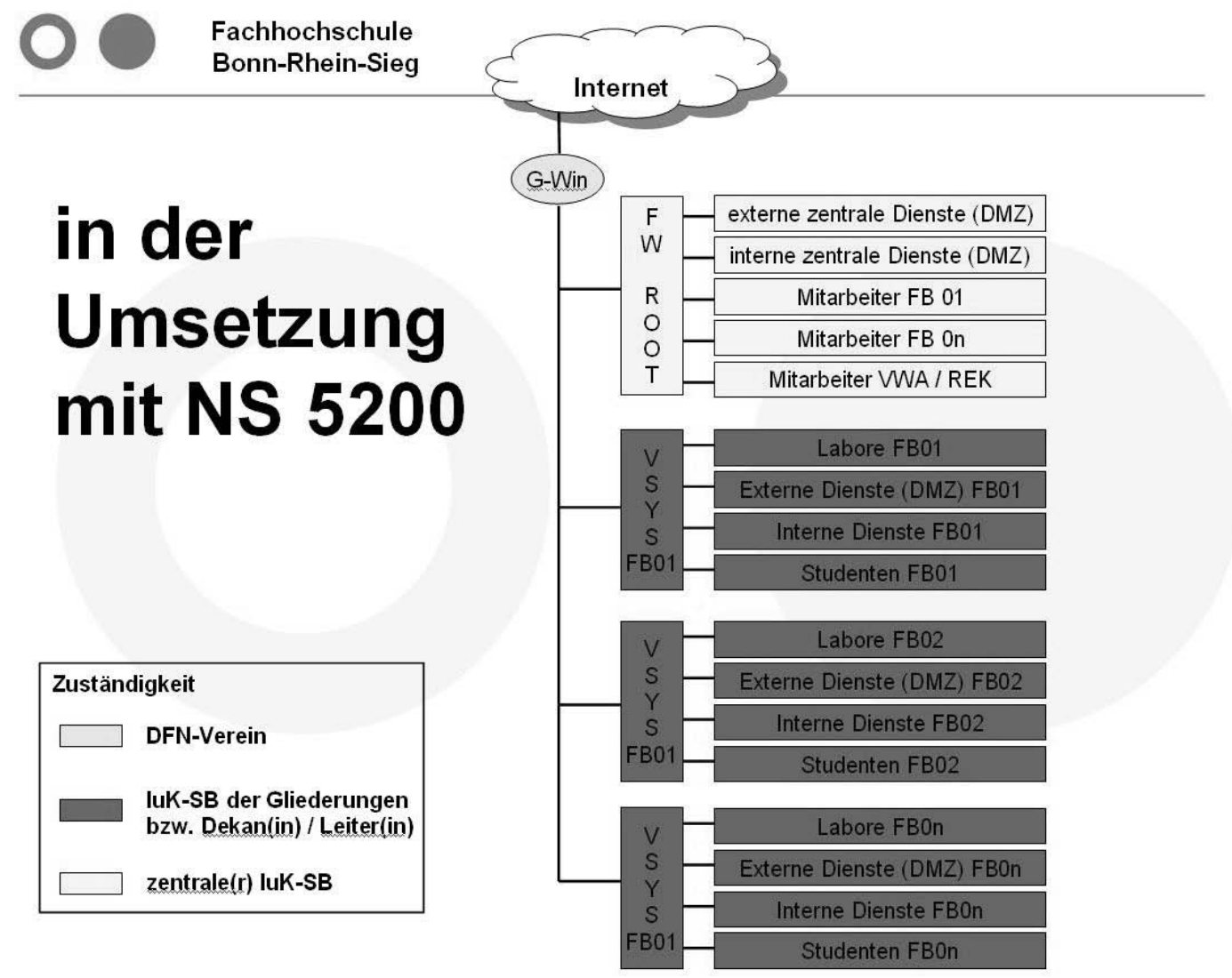

Anh. F Firewallumsetzung 